Kurzbeiträge

\title{
Heinz Bolsenkötter
}

\section{Modernisierung des Bilanzrechts - Auswirkungen auf öffentliche Unternehmen und Verwaltungen}

Anstaltsrecht; Bilanzrechtsmodernisierung; Eigenbetriebsrecht; Gemeindehaushaltsrecht; Haushaltsreform; Internationalisierung der Rechnungslegung

Vor dem Hintergrund internationaler Entwicklungen der Rechnungslegung von Unternehmen mit dem Bestreben weltweiter Harmonisierung steht auch die Bundesrepublik Deutschland vor einer weitgehenden Reform der einschlägigen Vorschriften im Dritten Buch des Handelsgesetzbuchs (HGB); diese Änderungen dürften die gewichtigsten seit denen des Bilanzrichtlinien-Gesetzes aus dem Jahre 1985 sein, mit denen seinerzeit für den EU-Raum eine Harmonisierung angestrebt wurde. Das bevorstehende Bilanzrechtsmodernisierungsgesetz (BilMoG) wird unmittelbar nur für Kaufleute und mit speziellen Vorschriften für Kapitalgesellschaften gelten; da aber kommunale Eigenbetriebe und rechtsfähige Anstalten z. T. Kaufmanns-Eigenschaft haben und da deren Rechnungslegung auch sonst weitgehend handelsrechtlichen Regelungen folgt und da ferner den Reformen der öffentlichen Haushalte allgemein das Dritte HGB-Buch als Referenzmodell zugrunde gelegt wurde, stellt sich die Frage nach unmittelbaren und mittelbaren Rückwirkungen des BilMoG auf die Rechnungslegung (und dabei auch auf die Haushalte) im öffentlichen Sektor. Dies ist nicht nur eine Frage des Geltungsbereichs und der Anwendung der anstehenden neuen Vorschriften, sondern wesentlich auch eine Frage nach dem sich ergebenden Handlungsbedarf, um Divergenzen im jeweils maßgeblichen Recht entgegenzuwirken bzw. sie demnächst zu beseitigen, die nicht sachlich begründet sind, sondern sich aus dem Ablauf des Gesetzgebungsverfahrens ergeben.

\section{Ansatzpunkte und Ziele des BilMoG}

Eine Bilanzrechtsmodernisierung wurde bereits durch die Vorgängerin der derzeitigen Bundesregierung eingeleitet; dieses Vorhaben wird auch grundsätzlich und mit seinen Tendenzen allgemein befürwortet. Gesetz wurde eine Übernahme der internationalen Rechnungslegungsregeln (International Financial Reporting Standards, IFRS) für die Konzernrechnungslegung kapitalmarktorientierter Unternehmen mit befreiender Wirkung hinsichtlich der sonst anzuwendenden diesbezüglichen HGB-Vorschriften (Bilanzrechtsreformgestz vom 10. Dezember 2004, BilReG). Die damit Bestandteil der deutschen 
Rechtsordnung gewordenen IFRS bilden naturgemäß wegen ihres Umfangs wie auch wegen ihrer Diktion einen Fremdkörper im deutschen Recht, wenn auch auf der Grundlage von EU-Verordnungen; wegen dieser europarechtlichen Grundlagen wird auch von EU-IFRS gesprochen.

Das BilMoG - so die Begründung zum Regierungsentwurf vom 21. Mai 2008 - soll eine moderate Annäherung an die IFRS bringen, ohne das bisherige System der Grundsätze ordnungsmäßiger Buchführung aufzugeben; die HGB-Bilanz soll - trotz deutlicher Stärkung ihrer Informationsfunktion - Grundlage der Ausschüttungsbemessung und der steuerlichen Gewinnermittlung (Grundsatz der Maßgeblichkeit der Handelsbilanz für die Steuerbilanz) bleiben. Erreicht werden soll damit eine dauerhafte und im Verhältnis zu den IFRS vollwertige, aber kostengünstigere und einfachere Alternative. Zum Beispiel wird auch der IFRS-Entwurf für kleine und mittlere Unternehmen mit seiner großen Regelungsdichte als zu komplex empfunden.

Der Regelungsgehalt des BilMoG bezieht sich auf die Übernahme zahlreicher einzelner Ansatz-, Ausweis- und Bewertungsregeln der IFRS und dabei tendenziell auch auf die Beseitigung von im deutschen Recht noch bestehenden Wahlrechten; daneben werden auch Vorgaben aus mehreren EU-Richtlinien - u. a. zur Erhöhung der Schwellenwerte der $\S \S 267$ und 293 HGB - umgesetzt. Das Gesetz wird als Artikelgesetz ausgestaltet, das außer HGB-Vorschriften zur Rechnungslegung auch Regelungen in korrespondierenden Gesetzen mit bilanzrechtlichen Implikationen ändert.

Diese Bestrebungen und Ansätze werden schon seit einigen Jahren intensiv erörtert und wohl im Allgemeinen auch positiv beurteilt; mit grundlegenden, die Tendenzen verschiebenden Änderungen dürfte im Gesetzgebungsverfahren wohl nicht mehr zu rechnen sein. Der Bundesrat hat schell reagiert und den Gesetzentwurf am 4. Juli 2008 beraten; in seiner Stellungnahme ${ }^{1}$ regt er einige Veränderungen/Verbesserungen an und empfiehlt in einigen wenigen Fällen tendenziell etwas Zurückhaltung bei den Reformen bzw. bei der Beseitigung von Wahlrechten. Die Konzeption des BilMoG wird dadurch aber nicht in Frage gestellt.

Das Anliegen dieses Beitrages ist es, auf die wesentlichen Neuerungen hinzuweisen und insbesondere frühzeitig auch auf die Auswirkungen auf den öffentlichen Sektor aufmerksam zu machen. Das BilMoG wird Kaufmannsrecht einschl. des Rechts der Personenhandelsgesellschaften und der Kapitalgesellschaften fortentwickeln, und dies auf der Ebene der Bundesgesetzgebung. In der Sache werden aber auch Einrichtungen, die nicht diesem Recht unterfallen, betroffen und es werden - wenn hier legislatorische Konsequenzen gezogen werden - auch die Zuständigkeiten der Bundesländer berührt. Als notwendig oder zweckmäßig zu erachtende Schritte sollten also nicht erst nach Verabschiedung des BilMoG durch den Bundesgesetzgeber angedacht und eingeleitet werden.

1 BRDrs 344/08 vom 4. Juli 08. 


\section{Wesentliche Reformmaßnahmen}

Im Hinblick auf die Thematik dieses Beitrags dürften folgende durch das BilMoG vorgesehene Änderungen besonders interessant $\operatorname{sein}^{2}$ (HGB-Hinweise in dieser Übersicht beziehen sich jeweils auf die im Entwurf vorgesehene Neufassung):

\section{Allgemeines}

- Der Grundsatz der wirtschaftlichen Betrachtungsweise (bei der Zurechnung von Vermögensgegenständen für die Bilanzierung) - der nicht neu ist - wird allgemein gültig in $\S 246$ Abs. $1 \mathrm{HGB}$ verankert.

- Die Grundsätze der Bilanzansatzstetigkeit (§ 246 Abs. 3 HGB) und der Bewertungsstetigkeit ( $\$ 252$ Abs. 1 Nr. 6 HGB) werden zwingend (als Muss-Vorschriften) vorgegeben.

- Der Grundsatz der sog. umgekehrten Maßgeblichkeit (§ 5 Abs. 1 S. 2 EStG, mehrere damit korrespondierende HGB-Regelungen) wird aufgehoben. Unter anderem entfallen künftig die Sonderposten mit Rücklagenteil; nach bisherigem Recht gebildete Sonderposten können beibehalten werden oder sind unmittelbar in die Gewinnrücklagen einzustellen (Art. 66 Abs. 1 EGHGB).

Bilanzierung, Ausweis

- Der Geschäfts- und Firmenwert gilt als Vermögensgegenstand (Fiktion, § 246 Abs. 1 S. 2 HGB); ist er derivativ, gilt Aktivierungspflicht, sonst (wie bisher) Aktivierungsverbot.

- Selbst geschaffene immaterielle Gegenstände des Anlagevermögens sind - von Ausnahmen abgesehen - aktivierungspflichtig und mit den Herstellungskosten der Entwicklungsphase (unter Ausschluss derjenigen der Forschungsphase) zu bewerten ( $\S 248,255$ Abs. 2a HGB); es gilt eine korrespondierende Ausschüttungssperre (§ 268 Abs. 8 HGB). Der Bundesrat schlägt Aktivierungswahlrecht vor.

- $\quad \mathrm{Zu}$ Handelszwecken erworbene Finanzinstrumente sind zum beizulegenden Zeitwert zu bewerten (§ 253 Abs. 1 S. 3 HGB; dies geht auf ein Mitgliedstaaten-Wahlrecht nach der EU-Fair-Value-Richtlinie zurück). Der Bundesrat empfiehlt, diese Regelung auf Finanzinstitute und Versicherungsunternehmen zu beschränken.

- Die bisherige Bilanzierungshilfe für Ingangsetzungs- und Erweiterungsaufwendungen (§ 269 HGB a. F.) wird aufgehoben.

- Nicht eingeforderte ausstehende Einlagen sind ebenso wie eigene Anteile ausschließlich auf der Passivseite vom „Gezeichneten Kapital“ abzusetzen (§ 272 Abs. 1, Abs. 1a, Abs. 4 HGB).

- Latente Steuern sind nach dem bilanzorientierten Konzept (für Deutschland wohl ohne nennenswerte Auswirkung gegenüber der bisherigen Methode) zu ermitteln;

2 Weitere, hier nicht im Einzelnen dargestellte Neuregelungen betreffen z. B. Befreiungen von Buchführungspflichten für kleine Einzelkaufleute (nach Bundesratsvorschlag auch für kleine Personenhandelsgesellschaften), Erhöhung der Schwellenwerte für die Anwendung bestimmter Vorschriften, Erweiterung der Pflichten für Anhang und Lagebericht sowie Neuerungen im Prüfungsrecht. 
auch aktive latente Steuern sind bilanzierungspflichtig (§ 274 HGB). Der Bundesrat schlägt Aktivierungswahlrecht vor.

- Das Wahlrecht zur Bildung von Aufwandsrückstellungen für unterlassene Instandhaltung u. ä. (§ 249 Abs. 1 S. 3, Abs. 2 HGB) wird aufgehoben; die bestehenden Rückstellungen können beibehalten werden oder sind unmittelbar in die Gewinnrücklage einzustellen (Art. 66 Abs. 1 EGHGB; diese letztere Variante ist auch methodisch neu).

Bewertung

- Bei der Rückstellungsbewertung sind künftige Preis- und Kostensteigerungen zu berücksichtigen (§ 253 Abs. 1 S. 2 HGB); Rückstellungen mit einer Laufzeit von mehr als einem Jahr sind mit dem ihrer Laufzeit entsprechenden durchschnittlichen Marktzinssatz der vergangenen sieben Geschäftsjahre (nach Bundesratsvorschlag mit dem Stichtagsmarktzinssatz) abzuzinsen. Rückstellungen für laufende Pensionen oder Anwartschaften auf Pensionen dürfen pauschal mit dem durchschnittlichen Marktzinssatz abgezinst werden, der sich bei einer angenommenen Laufzeit von 15 Jahren ergibt ( $\$ 253$ Abs. 2 HGB), dies gilt auch für Rentenverpflichtungen, bei denen eine Gegenleistung nicht mehr zu erwarten ist. Art. 28 Abs. 1 EGHGB mit dem Wahlrecht für Altzusagen und für mittelbare Verpflichtungen soll unverändert bestehen bleiben.

- Aufgehoben werden die Wahlrechte für Abschreibungen wegen künftiger Wertschwankungen sowie für Abschreibungen im Rahmen vernünftiger kaufmännischer Beurteilung ( $\$ 253$ Abs. 3 S. 3, Abs. 4 HGB); die außerplanmäßige Abschreibung bei nur vorübergehender Wertminderung wird auf Finanzanlagen beschränkt ( 253 Abs. 3 S. 4 HGB) und die Wertaufholung generell vorgeschrieben (§ 253 Abs. 5 S. 1 HGB).

- $\quad$ Einige der geplanten Neuregelungen sehen ausdrücklich die Bildung von Bewertungseinheiten ${ }^{3}$ vor:

- Eine Verrechnung von Vermögensgegenständen mit Schulden ist vorgeschrieben, soweit die Vermögensgegenstände allein zur Erfüllung der Schulden verwendet werden können ( 246 Abs. 2 HGB), also der allgemeinen Haftungsmasse des Unternehmens entzogen sind. Der Bundesrat empfiehlt, das nicht abgesicherte Risiko in einer Drohverlustrückstellung abzubilden.

- Zur Bildung von Bewertungseinheiten kommt es auch zwischen Vermögensgegenständen, Schulden, schwebenden Geschäften oder mit hoher Wahrscheinlichkeit vorgesehenen Transaktionen zum Ausgleich gegenläufiger Wertänderungen oder Zahlungsströme ( $254 \mathrm{HGB}$ ).

3 Den Terminus „Bewertungseinheit“ kennen die internationalen Standards zwar nicht, wohl aber vergleichbare Sachverhalte; verwiesen sei insbesondere auf die Sicherungsbilanzierung (hedge accounting). 
Hierzu sei auf die seit 2006 geltende Regelung des $\S 5$ Abs. 1a EStG hingewiesen, wonach die Ergebnisse der in der Handelsbilanz zur Absicherung finanzwirtschaftlicher Risiken gebildeten Bewertungseinheiten für die steuerliche Gewinnermittlung maßgeblich sind.

- $\quad$ Der handelsrechtliche Herstellungskostenbegriff wird an den des Steuerrechts angepasst, lässt also weniger Wahlrechte zu (§ 255 Abs. 2 HGB).

- Für die Währungsumrechnung von Vermögensgegenständen, Schulden, Rechnungsabgrenzungsposten, Aufwendungen und Erträgen gilt der Devisenkassakurs (§ 256a HGB).

Konzernrechnungslegung

Von den geplanten Änderungen zum Konzernabschluss seien hier lediglich genannt:

- Das Beteiligungskriterium des derzeitigen $\S 290$ Abs. 1 S. 1 HGB für den Begriff des Tochterunternehmens wird gestrichen; entscheidend wird also künftig ausschließlich die einheitliche Leitung sein.

- Die Kapitalkonsolidierung ist künftig nur noch nach der Neubewertungsmethode durchzuführen ( 301 Abs. 1 S. 2 HGB); die Buchwertmethode ist nicht mehr zulässig.

- Hingegen ist für die Behandlung assoziierter Unternehmen nur noch die Buchwertmethode vorgesehen ( $\$ 312$ Abs. 1 HGB); die derzeit erlaubte Kapitalanteilsmethode soll nicht mehr zulässig sein ${ }^{4}$.

- Die Aktiv- und Passivposten in Fremdwährungen sind zum Devisenkassakurs und die Posten der Gewinn- und Verlustrechnung zum Durchschnittskurs umzurechnen ( 308a HGB).

\section{Auswirkungen im öffentlichen Sektor}

Die Neuregelungen durch das BilMoG werden nur für Kaufleute allgemein (Erster Abschnitt des Dritten HGB-Buches) bzw. mit speziellen Regelungen für Kapitalgesellschaften gelten; dennoch ergeben sich u. E. erhebliche Auswirkungen im öffentlichen Sektor, die zu einigen grundlegenden Anschlussüberlegungen führen müssen. Dabei ist zu unterscheiden nach der Kernverwaltung der Gebietskörperschaften, die dem reformierten neuen Haushaltsrecht unterliegt, und ausgegliederten Einheiten, die gesondert Rechnung legen.

4 Die unterschiedliche Vorgehensweise bei der Vollkonsolidierung und der anteilmäßigen Konsolidierung mag überraschen, lässt sich aber begründen: Die Neubewertungsmethode bei der Vollkonsolidierung führt dazu, dass bei einem Anteilsbesitz von weniger als $100 \%$ die Anteile der Minderheiten an den stillen Reserven oder Lasten aufgedeckt werden. Die Regelung zur anteilmäßigen Konsolidierung assoziierter Unternehmen wird durch die Streichung der Kapitalanteilsmethode erheblich vereinfacht und lässt sich im Übrigen damit rechtfertigen, dass sich beide Methoden nur im Ausweis, nicht aber in der Sache unterscheiden; bei der Kapitalanteilsmethode ging der Firmenwert im Eigenkapital auf, bei der Buchwertmethode wird er gesondert gezeigt. 


\section{Anwendung von HGB-Vorschriften im Bereich des Haushaltsrechts}

Die bisherigen Haushaltsreformen orientieren sich - wenn auch mit zahlreichen vom HGB abweichenden Sonderregelungen und auch mit Unterschieden zwischen den Ländern - am Referenzmodell der HGB-Rechnungslegung nach dem für große Kapitalgesellschaften geltenden strengen Maßstab. Gesetzestechnisch wurde im Allgemeinen der Weg gewählt, HGB-Regelungen völlig neu ausformuliert mit den jeweils für angebracht gehaltenen Abweichungen/Ergänzungen in das Kommunalrecht/Landesrecht einzufügen; pauschale, umfassende Verweise auf Handelsrecht wurden also weitgehend vermieden. Wo im Einzelfall, also mit sachlich begrenztem Geltungsbereich (im Wesentlichen für den Gesamtabschluss bzw. die „Konzernrechnungslegung“) auf Handelsrecht verwiesen wird, geschieht dies regelmäßig mit dem Hinweis darauf, dass die jeweils gemeinten HGB-Regelungen in der zur Zeit der Schaffung der Verweisvorschrift geltenden Fassung anzuwenden sind; verwiesen wird also ausdrücklich statisch. Als Beispiel sei $§ 49$ Abs. 4 GemHVO NRW genannt, wonach sich HGB-Verweise ,in diesem Abschnitt“ (betrifft den Gesamtabschluss) auf das HGB in der zuletzt am 24. August 2002 geänderten Fassung beziehen. Die Verweise im Haushaltsrecht sind also weder umfassend noch können sie dynamisch - in der jeweils gültigen Fassung von HGB-Regelungen - interpretiert werden $^{5}$; im Einzelnen ist das naturgemäß nach dem jeweiligen Landesrecht zu überprüfen.

Nach Inkrafttreten des BilMoG (voraussichtlich 2009; die derzeit noch geltenden vom BilMoG betroffenen Regelungen sollen letztmalig auf das vor dem 1. Januar 2009 beginnende Geschäftjahr angewendet werden, Art. 66 Abs. 9 E-EG HGB) wird sich also die unbefriedigende Situation ergeben, dass die Rechnungslegung im unternehmerischen, vom Handelsrecht erfassten Bereich - auch bei den betroffenen Unternehmen von Gebietskörperschaften - mit einem großen Schritt modernisiert und weitgehend internationalen Regeln und Grundsätzen angenähert sein wird; über EU-Verordnungen und das deutsche Bilanzrechtsreformgesetz sind diese Regeln übrigens auch bereits Bestandteil der deutschen Rechtsordnung geworden, wenn auch derzeit noch nicht mit allgemeiner Geltung (jedenfalls aber für Konzernabschlüsse kapitalmarktorientierter Unternehmen). Die legislatorisch bereits realisierten Reformen von Rechnungs- und Haushaltswesen im öffentlichen Sektor hingegen folgen dann demnächst einem inzwischen z. T. überholten Modell, wenn bzw. solange die jeweiligen Gesetzgeber nicht erneut tätig werden, solange also derzeit geltendes Recht bestehen bleibt.

Dabei geht es nicht nur um die Frage, ob man dies wegen der (weiteren) Beeinträchtigung der Vergleichbarkeit bedauert; inhaltlich sind manche der BilMoG-Neuerungen (z. B. die Aktivierung selbst erstellten immateriellen Anlagevermögens und die Abzinsung langfristiger Rückstellungen) auch für den öffentlichen Sektor zu begrüßen, während das Verbot von Aufwandsrückstellungen - mit denen ein Instandhaltungsstau wenigstens teilweise sichtbar gemacht werden konnte - sicherlich von vielen bedauert wird,

5 Ein einzelnes Gegenbeispiel ist wohl $\S 51$ Abs. 3 GemHVO NRW. Der dortige Verweis auf den Rechnungslegungsstandard zur Kapitalflussrechnung (DRS 2) muss wohl dynamisch verstanden werden. 
wegen der international gebräuchlichen Handhabung aber wohl nicht zu vermeiden ist. Es sind vielmehr auch handfeste, gravierende Probleme zu befürchten.

Zur öffentlichen Rechnungslegung gehört - nach Ablauf von Übergangsfristen - regelmäßig auch ein Gesamtabschluss (,Konzernabschluss“), in den neben der Kernverwaltung der Gebietskörperschaften auch die ausgegliederten Einheiten nach Konsolidierungsregeln einzubeziehen sind. Zu den ausgegliederten Einheiten gehören aber viele, die mit ihren Jahresabschlüssen zwingend dem HGB und damit demnächst dem HGB in der BilMoG-Fassung unterliegen; auch werden durch das BilMoG die Konsolidierungsregeln teilweise geändert. Hier können sich also demnächst Konflikte hinsichtlich der Anwendung divergierender Rechtsvorschriften bzw. deren Harmonisierung durch einheitliche Bilanzierungs- und Bewertungsregeln (das könnten dann wohl nur die nach altem Recht sein) ergeben; insoweit zeigt sich also ein erneuter baldiger Reformbedarf. Man mag daran denken, die aufgrund von Revisionsklauseln (z. B. § 10 NKFEG NRW) ohnehin vorgeschriebene Überprüfung der Auswirkungen der haushaltsrechtlichen Reformgesetze mit einer Anpassung an die neuen HGB-Regelungen zu verbinden, jedenfalls dort, wo dies zeitnah geschehen kann.

$\mathrm{Zu}$ denken ist auch daran, dass derzeit noch nicht alle Bundesländer das Gemeindehaushaltsrecht reformiert haben und dass großenteils die Neugestaltung des Länderhaushaltsrechts ebenso wie die des Bundeshaushaltsrechts noch aussteht. Wenn und soweit auch hier die HGB-Rechnungslegung als Referenzmodell dienen wird - was allerdings teilweise umstritten ist - wäre es wohl kaum vertretbar, diese Reformen an überholte HGB-Vorschriften anzulehnen, nur weil diese in einigen Ländern bei den Reformen bereits als Referenzmodell gedient haben.

\section{Anwendung von HGB-Vorschriften auf kommunale Eigenbetriebe und Anstalten}

Kommunale Eigenbetriebe und rechtsfähige Anstalten mit Kaufmannseigenschaft unterliegen den Regelungen des Ersten Abschnitts des Dritten Buches des HGB (§§ 238-263 HGB) soweit nicht abweichende landesrechtliche Vorschriften eingreifen ( $\$ 263 \mathrm{HGB}$ ); insoweit wird das BilMoG auch hier unmittelbar wirksam. Die Landesgesetzgeber haben die Möglichkeit des $\S 263$ HGB bisher nur zurückhaltend genutzt, also das Haushaltsrecht weitestgehend übernommen ${ }^{6}$.

Im Übrigen wird im kommunalen Eigenbetriebs- und Anstaltsrecht recht ausgiebig auf die handelsrechtlichen Vorschriften für (große) Kapitalgesellschaften verwiesen, und zwar ohne den Hinweis auf eine bestimmte HGB-Fassung ${ }^{7}$; kommunalrechtliche Sonderregelungen (die hier nicht der Rechtfertigung durch $\S 263$ HGB bedürfen) gibt es auch hier, z. B. Jahresabschluss-Formblätter. Diese HGB-Verweise wurden bisher allgemein dynamisch interpretiert ${ }^{8}$; das erschien wohl auch deshalb vertretbar, weil sowohl die

6 S. hierzu die Übersicht bei Bolsenkötter/Dau/Zuschlag 2004, Rn W 281.

7 S. Bolsenkötter/Dau/Zuschlag 2004, Rn W 278-282.

8 S. Bolsenkötter/Dau/Zuschlag 2004, Rn W 781. 
Rechnungslegung nach dem HGB als auch die - zeitlich anschließend realisierte - nach kommunalem Eigenbetriebs- und Anstaltsrecht geschaffene auf das BilanzrichtlinienGesetz vom 19. Dezember 1985 zurückgingen und weil die HGB-Rechnungslegung seitdem nicht grundlegend umgestaltet wurde ${ }^{9}$. Ob eine dynamische Interpretation der Verweise auch nach so umfänglichen und gewichtigen Änderungen, wie sie das BilMoG bringen wird, juristisch und das heißt verfassungsrechtlich vertretbar ist, muss allerdings ernsthaft bezweifelt werden, so dass es sogar innerhalb eines Betriebes/einer Anstalt zu der Situation kommen kann, dass Handelsrecht nach unterschiedlichem Stand der Gesetzgebung anzuwenden ist, nämlich auf Grund der Kaufmannseigenschaft anzuwendendes neues Recht und auf Grund von Verweisen anzuwendendes altes Recht. Damit ergibt sich also auch hier - und zwar in mehrfacher Hinsicht - ein erheblicher Novellierungsbedarf zur Wahrung möglichster Rechtseinheitlichkeit.

\section{Schlussfolgerungen}

Dass im Gefolge des BilMoG auf Grund der vorstehend geschilderten unmittelbaren und mittelbaren Auswirkungen im öffentlichen Sektor erheblicher Handlungsbedarf für eine Folgegesetzgebung besteht, dürfte angesichts der oben geschilderten Situation wohl kaum bezweifelt werden. Es ist auch dringend erwünscht, dass die dann anstehenden legislatorischen Maßnahmen möglichst abgestimmt realisiert worden.

Den hier hauptsächlich betroffenen Regelungen lagen im kommunalen Bereich bisher zwei Empfehlungen zugrunde:

- Arbeitskreis III „Kommunale Angelegenheiten“ der Innenministerkonferenz der Länder, Musterentwurf einer Eigenbetriebsverordnung in der an das Bilanzrichtlinien-Gesetz angepassten Fassung vom 14. März 1986 - Musterentwurf $1986^{10}$;

- Arbeitskreis III „Kommunale Angelegenheiten“ der Innenministerkonferenz der Länder,

I Erläuterungen zum Leittext für eine Gemeindehaushaltsverordnung für ein doppisches Haushalts- und Rechnungswesen

II Leittext Gemeindehaushaltsverordnung für ein doppisches Haushalts- und Rechnungswesen

Den 8./9. Oktober 2003

Erarbeitet vom Unterausschuss „Reform des Gemeindehaushaltsrechts“ des AK III und verabschiedet am 17./18. Juni 2003.

Es bietet sich an, bei diesen Arbeiten anzuknüpfen und sie unter Berücksichtigung der durch das BilMoG herbeigeführten HGB-Änderungen fortzuentwickeln. Darin kann sogar eine Chance liegen, denn die bisherigen Haushaltsreformen sind nicht im gleichen Maße harmonisiert, wie das etwa zwei Jahrzehnte zuvor mit dem EigVO-Musterentwurf

9 Zum Teil allerdings wird die verfassungsrechtliche Problematik dynamischer Verweise kritisch gesehen; s. Ossenbühl 1967; Stern 1980, S. 635. Unzulässige dynamische Verweise können auch statisch umgedeutet werden (BVerfG-Urt. vom 1. März 1978).

10 Im Wortlaut mit Anmerkungen abgedruckt in: Bolsenkötter/Dau/Zuschlag 2004, S. 632-648. 
gelungen war. Die u. E. notwendigen Anpassungen können also mit einer anzustrebenden stärkeren Abstimmung und Vereinheitlichung verbunden werden. Zu hoffen bleibt, dass das auch die noch ausstehenden Reformen in diesem Sinne beeinflussen wird.

\section{Literaturverzeichnis}

Bundesregierung, Entwurf eines Gesetzes zur Modernisierung des Bilanzrechts (Bilanzrechtsmodernisierungsgesetz-BilMoG) vom 21. Mai 2008.

Bundesrat, Stellungnahme zum Entwurf eines Gesetzes zur Modernisierung des Bilanzrechts (Bilanzrechtsmodernisierungsgesetz - BilMoG) vom 4. Juli 2008, BRDrs. 344/08 (Beschluss).

Arbeitskreis III „Kommunale Angelegenheiten“ der Ständigen Konferenz der Innenminister und -senatoren der Länder, Musterentwurf einer Eigenbetriebsverordnung in der an das Bilanzrichtlinien-Gesetz angepassten Fassung vom 14. März 1986 - Musterentwurf 1986.

Arbeitskreis III „Kommunale Angelegenheiten“ der Ständigen Konferenz der Innenminister und -senatoren der Länder,

I Erläuterungen zum Leittext für eine Gemeindehaushaltsverordnung für ein doppisches Haushalts- und Rechnungswesen

II Leittext Gemeindehaushaltsverordnung für ein doppisches Haushalts- und Rechnungswesen

Den 8./9. Oktober 2003

Erarbeitet vom Unterausschuss „Reform des Gemeindehaushaltsrechts“ des AK III und verabschiedet am 17./18. Juni 2003.

Bundesverfassungsgericht, Urteil vom 1. März 1978, in: BVerfGE 47, S. 285, Neue Juristische Wochenschrift, 31. Jg., Heft 30, S. 1475.

Bolsenkötter, Heinz, Horst Dau und Eckhard Zuschlag (2004), Gemeindliche Eigenbetriebe und Anstalten - Länderübergreifende Darstellung, 5. Aufl., Stuttgart.

Ossenbühl, Fritz (1964), Die verfassungsmäßige Zulässigkeit der Verweisung als Mittel der Gesetzgebungstechnik, in: Deutsches Verwaltungsblatt, 82. Jg., Heft 10, S. 401.

Stern, Klaus (1980), Das Staatsrecht der Bundesrepublik Deutschland, Bd. II, München. 